SLAC-PUB-9656

February 2003

\title{
Precision Measurement of the Proton and Deuteron Spin Structure Functions $\mathrm{g}_{2}$. ${ }^{*}$
}

\author{
Stephen Rock For the E155 Collaboration \\ University of Massachusetts, Amherst, Massachusetts, USA 01003
}

\begin{abstract}
We measured the spin structure functions $g_{2}^{p}$ and $g_{2}^{d}$ in the range $0.02 \leq$ $x \leq 0.8$ and $0.7 \leq Q^{2} \leq 20 \mathrm{GeV}^{2}$ by scattering 29.1 and $32.3 \mathrm{GeV}$ longitudinally polarized electrons from transversely polarized $\mathrm{NH}_{3}$ and ${ }^{6} \mathrm{LiD}$ targets. $g_{2}$ approximately follows the twist-2 Wandzura-Wilczek calculation. The twist-3 reduced matrix elements $d_{2}^{p}$ and $d_{2}^{n}$ are less than two standard deviations from zero. The data are inconsistent with the BurkhardtCottingham sum rule if there is no pathological behavior as $x \rightarrow 0$. The Efremov-Leader-Teryaev integral is consistent with zero.
\end{abstract}

PACS numbers: 13.60.Hb, 13.88.+e, 24.70.+s, 25.30.Fj

\section{Introduction}

The deep inelastic spin structure functions of the nucleons, $g_{1}\left(x, Q^{2}\right)$ and $g_{2}\left(x, Q^{2}\right)$, depend on the spin distribution of the partons and their correlations. The function $g_{1}$ can be primarily understood in terms of the quark parton model (QPM) and perturbative QCD with higher twist terms at low $Q^{2}$. The function $g_{2}$ is of particular interest since it has contributions from quark-gluon correlations and other higher twist terms at leading order in $Q^{2}$ which cannot be described perturbatively. By interpreting $g_{2}$ using the operator product expansion (OPE) $[1,2]$, it is possible to study contributions to the nucleon spin structure beyond the simple QPM.

The structure function $g_{2}$ can be written[3]:

$$
\begin{aligned}
g_{2}\left(x, Q^{2}\right) & =g_{2}^{W W}\left(x, Q^{2}\right)-\int_{x}^{1} \frac{\partial}{\partial y}\left(\frac{m}{M} h_{T}\left(y, Q^{2}\right)+\xi\left(y, Q^{2}\right)\right) \frac{d y}{y}, \\
g_{2}^{W W}\left(x, Q^{2}\right) & =-g_{1}\left(x, Q^{2}\right)+\int_{x}^{1} \frac{g_{1}\left(y, Q^{2}\right)}{y} d y,
\end{aligned}
$$

\footnotetext{
* Presented at DIS 2002.
}

Presented at the 10th International Workshop On Deep Inelastic Scattering (DIS2002), Cracow, Poland, 4/30/2002 - 5/4/2002

Work supported in part by the Department of Energy contract DE-AC03-76SF00515. Stanford Linear Accelerator Center, Stanford, University, Stanford, CA 94309 
where $x$ is the Bjorken scaling variable and $Q^{2}$ is the absolute value of the virtual photon four-momentum squared. The twist-2 term $g_{2}^{W W}$ was derived by Wandzura and Wilczek[4]. The transverse polarization density $h_{T}\left(x, Q^{2}\right)$ is a twist-2 contribution $[3,5]$. which is suppressed by the ratio of the quark to nucleon masses $m / M[5]$. The twist-3 part $(\xi)$ comes from quark-gluon correlations and is the main focus of our study.

Electron beams with energies of 29.1 and $32.3 \mathrm{GeV}$ and longitudinal polarizations of $P_{b}=(83.2 \pm 3.0) \%$ struck approximately transversely polarized $\mathrm{NH}_{3}$ (average polarization $\left.\left\langle P_{t}\right\rangle=0.70\right)$ or $\left.{ }^{6} \mathrm{LiD}\left(<P_{t}\right\rangle=0.22\right)$ targets. The beam helicity was randomly chosen pulse by pulse. Scattered electrons were detected in three independent spectrometers centered at $2.75^{\circ}$, $5.5^{\circ}$, and $10.5^{\circ}$. We determined $g_{2}$ from the experimental asymmetry by correcting for beam and target polarization, dilution factor, hadron contamination, pair symetric background, electro-weak asymmetry, radiative corrections and the contribution of $g_{1}$. The structure functions for $\mathrm{p}, \mathrm{d}$, and $\mathrm{n}$ are related by $g_{2}^{d}=\left(g_{2}^{p}+g_{2}^{n}\right)\left(1-1.5 \omega_{D}\right) / 2$, where $\omega_{D}=0.05$, the fraction of $\mathrm{D}$-wave in the deuteron wave function. A more complete description of the experimental method and results can be found in Ref. [6].

The data cover the kinematic range $0.02 \leq x \leq 0.8$ and $0.7 \leq Q^{2} \leq$ $20 \mathrm{GeV}^{2}$ with an average $Q^{2}$ of $5 \mathrm{GeV}^{2}$. Figure 1 shows the values of $x g_{2}$ as a function of $Q^{2}$ for several values of $x$ along with results from SLAC experiments E143 and E155. The data approximately follow the $Q^{2}$ dependence of $g_{2}^{W W}$ (solid curve), although for the proton, the data points are lower than $g_{2}^{W W}$ at low and intermediate $x$ and higher at high $x$. The predictions of Stratmann[7] are closer to the data.

To get average values at the average $Q^{2}$ for each $x$ bin we used the $Q^{2}$ dependence of $g_{2}^{W W}: g_{2}\left(Q_{\text {avg }}^{2}\right)=g_{2}\left(Q_{\text {exp }}^{2}\right)-g_{2}^{W W}\left(Q_{\text {exp }}^{2}\right)+g_{2}^{W W}\left(Q_{\text {avg }}^{2}\right)$. Figure 2 shows the averaged $x g_{2}$ of this experiment along with $x g_{2}^{W W}$ calculated using our parameterization of $g_{1}$. The combined new data for $\mathrm{p}$ disagree with $g_{2}^{W W}$ with a $\chi^{2} /$ dof of 3.1 for 10 degrees of freedom. For d the new data agree with $g_{2}^{W W}$ with a $\chi^{2} /$ dof of 1.2 for 10 dof. The data for $g_{2}^{p}$ are also inconsistent with zero $\left(\chi^{2} /\right.$ dof=15.5) while $g_{2}^{d}$ differs from zero only at $x \sim 0.4$. Also shown in Fig. 2 is the Bag Model calculation of Stratmann[7] which is in good agreement with the data, a chiral soliton model calculation[13] which is too negative at $x \sim 0.4$ and the Bag Model calculation of Song[5] which is in clear disagreement with the data.

Using the OPE, the moments of $g_{1}$ and $g_{2}$ for even $n \geq 2$ at fixed $Q^{2}$ can be related to twist- 3 reduced matrix element, $d_{n}$, and higher twist terms which are suppressed by powers of $1 / Q$. Neglecting quark mass terms we 


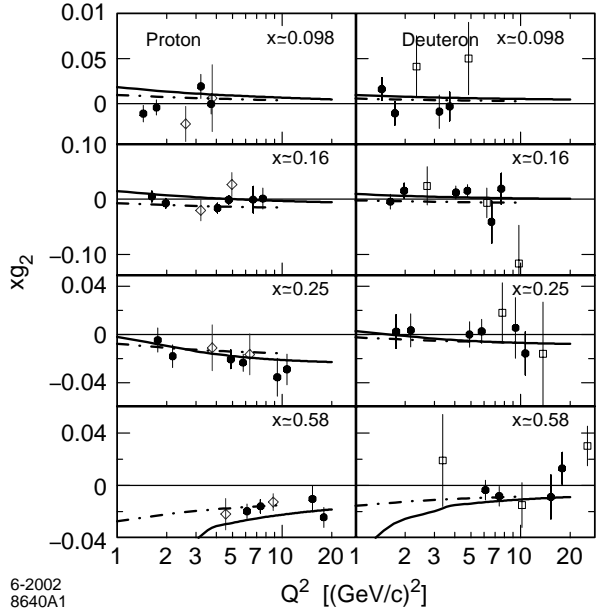

Fig. 1. The structure function $x g_{2}$ for the proton and deuteron as a function of $Q^{2}$ for selected values of $x$. Data are for this experiment (solid), E143 (open diamond) and E155 (open square). The errors are statistical; the systematic errors are negligible. The curves show $x g_{2}^{W W}$ (solid) and the bag model calculation of Stratmann[7] (dash-dot).

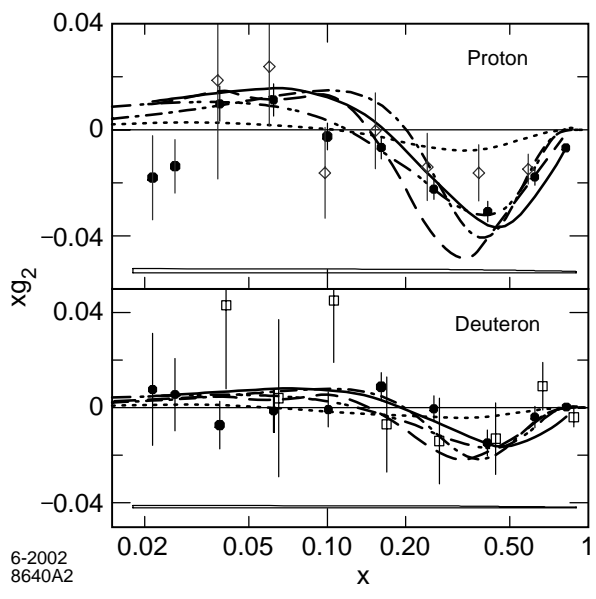

Fig. 2. The structure function $x g_{2}$ for all spectrometers combined (solid circle) and data from E143 (open diamond) and E155 (open square). The errors are statistical; the systematic errors are shown at the bottom. Also shown is our twist- $2 \mathrm{~g}_{2}^{W W}$ at the average $Q^{2}$ of this experiment at each value of $x$ (solid line). The curves are the bag model calculations of Stratmann[7] (dash-dot) and Song[5] (dot) and the chiral soliton models of Weigel and Gamberg[13] (short dash) and Wakamatsu[14] (long dash).

find that:

$d_{n}=2 \int_{0}^{1} d x x^{n}\left[\frac{n+1}{n} g_{2}\left(x, Q^{2}\right)+g_{1}\left(x, Q^{2}\right)\right]=2 \frac{n+1}{n} \int_{0}^{1} d x x^{n}\left(g_{2}-g_{2}^{W W}\right)$.

The matrix element $d_{n}$ measures deviations of $g_{2}$ from the twist- $2 g_{2}^{W W}$ term. Note that some authors $[2,12]$ define $d_{n}$ with an additional factor of two. The part of the $d_{2}$ integral for $x$ below the measured region was assumed to be zero because of the $x^{2}$ suppression. Because $g_{2}$ is small at high $x$, that contribution was negligible. We obtained values of $d_{2}^{p}=0.0025 \pm$ $0.0016 \pm 0.0010$ and $d_{2}^{d}=0.0054 \pm 0.0023 \pm 0.0005$ at an average $Q^{2}$ of 5 $\mathrm{GeV}^{2}$. We combined these results with those from previous SLAC experiments on the neutron, proton and deuteron to obtained average values 


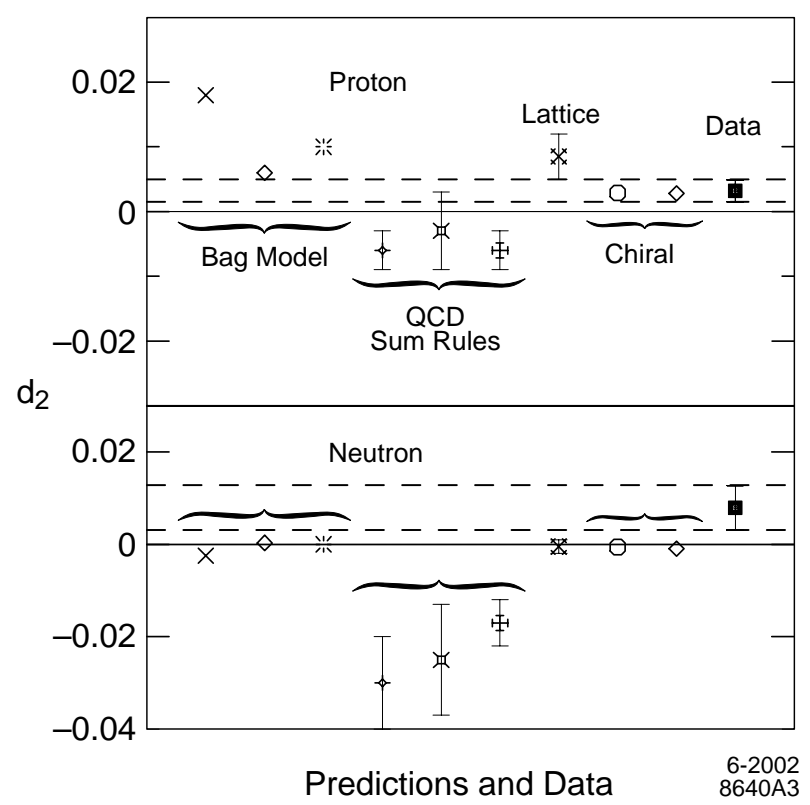

Fig. 3. The twist-3 matrix element $d_{2}$ for the proton and neutron. Also shown are theoretical model values from left to right: Bag Models[5, 7, 8], QCD Sum Rules $[9,10,11]$, Lattice QCD [12] and Chiral Soliton Models[13, 14]. The region between the dashed lines indicates the experimental errors.

$d_{2}^{p}=0.0032 \pm 0.0017$ and $d_{2}^{n}=0.0079 \pm 0.0048$. These are consistent with zero (no twist-3) to within 2 standard deviations.

Figure 3 shows the experimental values of $d_{2}$ for proton and neutron with their error, plotted along with theoretical models from left to right: Bag Models (Song[5], Stratmann[7], and Ji[8]); sum rules (Stein[9], BBK[10], Ehrnsperger[11]); lattice QCD calculations $\left(Q^{2}=5 \mathrm{GeV}^{2}, \beta=6.4\right)[12]$; and chiral soliton models $[13,14]$. The lattice and chiral calculations are in good agreement with the proton data and two standard deviations below the neutron data. The sum rule calculations are significantly lower than the data. The Non Singlet $=3 \cdot\left(d_{2}^{p}-d_{2}^{n}\right)=-0.0141 \pm 0.0170$ is consistent with an instanton vacuum calculation of $\sim 0.001$ [15].

The Burkhardt-Cottingham sum rule[16] for $g_{2}$ at large $Q^{2}, \int_{0}^{1} g_{2}(x) d x=$ 0 , was derived from virtual Compton scattering dispersion relations. It does not follow from the OPE since $n=0$. Its validity depends on the lack of singularities for $g_{2}$ at $x=0$. We evaluated the Burkhardt-Cottingham integral in the measured region of $0.02 \leq x \leq 0.8$ at $Q^{2}=5 \mathrm{GeV}^{2}$. The results for the proton and deuteron are $-0.044 \pm 0.008 \pm 0.003$ and $-0.008 \pm 0.012 \pm 0.002$ respectively. Averaging with the E143 and E155 re- 
sults which cover a slightly more restrictive $x$ range gives $-0.042 \pm 0.008$ and $-0.006 \pm 0.011$. This does not represent a conclusive test of the sum rule because the behavior of $g_{2}$ as $x \rightarrow 0$ is not known. However, if we assume that $g_{2}=g_{2}^{W W}$ for $x<0.02$, and use the relation $\int_{0}^{x} g_{2}^{W W}(y) d y=$

$x\left[g_{2}^{W W}(x)+g_{1}(x)\right]$, there is an additional contribution of $0.020(0.004)$ for the proton (deuteron).

The Efremov-Leader-Teryaev (ELT) sum rule[17] is for valance quarks.

It takes the form: $\int_{0}^{1} x\left[g_{1}^{p}(x)+2 g_{2}^{p}(x)-g_{1}^{n}(x)-2 g_{2}^{n}(x)\right] d x=0$. if the sea quarks are the same in protons and neutrons. We evaluated this ELT integral in the measured region using our $g_{2}$ data and the fit to $g_{1}$. The result at $Q^{2}=5 \mathrm{GeV}^{2}$ is $-0.013 \pm 0.008 \pm 0.002$, which is consistent with the expected value of zero. Including the data of E143 and E155 leads to $-0.011 \pm 0.008$. The extrapolation to $x=0$ is not known, but is suppressed by a factor of $x$.

\section{REFERENCES}

[1] E. Shuryak and A. Vainshtein, Nuc. Phys. B 201, 141 (1982).

[2] R. Jaffe and X. Ji, Phys. Rev. D 43, 724 (1991).

[3] J. L. Cortes, B. Pire and J. P. Ralston, Z. Phys. C 55, 409 (1992).

[4] S. Wandzura and F. Wilczek, Phys. Lett. B 72, 195 (1977).

[5] X. Song, Phys. Rev. D 54, 1955 (1996).

[6] P. Anthony et al., SLAC-PUB-8813 (hep-ex/0204028).

[7] M. Stratmann, Z. Phys. C 60, 763 (1993).

[8] X. Ji and P. Unrau, Phys. Lett. B 333, 228 (1994).

[9] E. Stein et al., Phys. Lett. B 343, 369 (1995).

[10] I. Balitsky, V. Braun and A. Kolesnichenko, Phys. Lett. B 242, 245 (1990); 318, 648 (1993) (Erratum).

[11] B. Ehrnsperger and A. Schafer, Phys. Rev. D 52, 2709 (1995).

[12] M. Göckeler et al., Phys. Rev. D 63, 074506 (2001).

[13] H. Weigel and L. Gamberg, Nucl. Phys. A 680, 48 (2000).

[14] M. Wakamatsu, Phys. Lett. B 487, 118 (2000).

[15] J. Balla, M.V. Polyakov, and C. Weiss, Nucl. Phys. B 510, 327 (1998).

[16] H. Burkhardt and W. N. Cottingham, Ann. Phys. 56, 453 (1970).

[17] A. V. Efremov, O. V. Teryaev and E. Leader, Phys. Rev. D55, 4307 (1997). 